\title{
Enhancing Network Management via NFV, MEC, Cloud Computing and Cognitive Features: The "5G ESSENCE" Modern Architectural Approach
}

Ioannis P. Chochliouros ${ }^{1}$, Anastasia S. Spiliopoulou ${ }^{1}$, Anastasios Kourtis 2 , Ioannis Giannoulakis ${ }^{2}$, Michail-Alexandros Kourtis ${ }^{2}$, Emmanouil Kafetzakis ${ }^{3}$,

Eirini Vasilaki ${ }^{1}$, Marinos Agapiou ${ }^{4}$ and Mike Iosifidis ${ }^{5}$

${ }^{1}$ Hellenic Telecommunications Organization (OTE) S.A., 99, Kifissias Avenue, GR 15124, Maroussi, Athens, Greece \{ichochliouros, evasilaki\}@oteresearch.gr aspiliopoul@ote.gr

${ }^{2}$ National Centre for Scientific Research "Demokritos", Patriarchou Gregoriou Street, Aghia Paraskevi, GR 15310, Athens, Greece \{kourtis, giannoul, akis.kourtis\}@iit.demokritos.gr

${ }^{3}$ ORION Innovations Private Company, 43, Ameinokleous Street, GR 11744, Athens, Greece mkafetz@orioninnovations.gr

${ }^{4}$ National \& Kapodistrian University of Athens, 6, Panepistimiopolis, GR 15784, Ilissia-Athens, Greece sdi1400002@di.uoa.gr

${ }^{5}$ CLEMIC Services S.A., 55, Salaminos Street, GR 15124, Maroussi, Athens, Greece mike@clemic.net

\begin{abstract}
The paper work presents the essential architectural approach that has been proposed in the framework of the modern "5G-ESSENCE" EU-funded project, intending to develop a suitable ecosystem for serving real-life use cases associated to vertical industries and built on the pillars of network functions virtualization (NFV), mobile-edge computing (MEC) capabilities, cognitive network management and appropriate use of small cells. Apart from identifying innovative features and options for enabling service deployment, the work focuses on the fundamental 5G ESSENCE-based architecture with description of the corresponding modules and their capabilities. The current approach comes as a mature continuity of previous efforts and/or related findings in the 5G-PPP context (mainly from the SESAME research project), but it purely concentrates upon providing tools for a robust and agile network management.
\end{abstract}

Keywords: $5 \mathrm{G}$, cognitive management, edge cloud computing, Mobile Edge Computing (MEC), Network Functions Virtualization (NFV), network slicing, Small Cell (SC), Virtual Network Function (VNF). 


\section{Introduction: The 5G ESSENCE Context in the 5G Era}

Today, Internet and communication networks are "critical" tools for most areas and sectors of our modern societies and economies as they are transforming our world; actually, these networks constitute fundamental "pillars" for any evolutionary process supporting effort for growth and development. According to recent market trends [1] as well as to actual European policy measures and/or related initiatives [2], it is assessed that the communication networks and the wider modern services/facilities environment of the year 2020 will be "enormously richer and much more complex than that of today". The expected diversity of new (personal and professional) usages results in new requirements on availability, latency, reliability, trustworthiness and security. These chances are so expected to take place within the forthcoming "fifth generation" -or $5 G$ - of telecoms systems, that will be the most critical building block of our "digital society" in the next decade; $5 \mathrm{G}$ will not only be an evolution of mobile broadband networks but will bring new unique network and service capabilities, creating a sustainable and scalable technology but also a proper ecosystem for technical and business innovation ([3], [4]). Among current 5G's priorities is also to incorporate advanced automation, autonomicity and cognitive management features to advance operators' efficiency. This can also have a positive impact on the broader competitiveness of the European ICT industry. Furthermore, 5G can also support and enhance the convergence between fixed and mobile networking services with the related development of core and transport networks. 5G can "integrate networking, computing and storage resources into one programmable and unified infrastructure", which can be customized according to the interests of multiple costumers. As a consequence, the simultaneous "inclusion" of modern features (such as of virtualisation and of software-based network functionalities) in communications infrastructures is expected to support the corresponding transitional process via further strengthening network flexibility and reactivity [5]. Market "actors" (network operators and service providers, manufacturers, SMEs, end-users, etc.) are expected to be strongly involved in such processes; this will "redefine" existing value chains and reform roles and/or relationships between market "players", whilst creating new opportunities for novelty and investments. $5 \mathrm{G}$ is also expected to drastically reduce total cost of ownership of the infrastructure, on one hand, and the service creation and deployment times, on the other.

During 5G-PPP Phase-1, the ongoing SESAME project [6] evolved the small cell (SC) concept by integrating processing power (i.e., a low-cost micro-server) and by enabling the execution of applications and network services, in accordance to the Mobile Edge Computing (MEC) paradigm [7]. It also provides network intelligence and applications by leveraging the Network Function Virtualisation (NFV) concept [8]. The SESAME platform consists of one or more clusters of "Cloud - Enabled" Small Cells (CESCs), which are devices that include both the processing power platform and the small cell unit. CESCs can be deployed at low- and medium-scale venues and support multiple network operators (i.e.: multitenancy) and further, network services and applications at the edge of the network. In this context, SESAME has developed several small cell related functions as Virtualised Network Functions (VNFs), such as the GPRS Tunneling Protocol (GTP) en-/de-capsulation of data packets. Also SESAME has demonstrated so far that some network-related 
functions (such as content caching, firewalls and monitoring) perform adequately well when running as VNFs in the developed micro-server infrastructure (coined as "Light Data Centre" -Light DC-).

The 5G ESSENCE project [9] leverages results from the prior SESAME project, as well as from other 5G-PPP Phase-1 projects (mainly COHERENT [10], SPEED 5G [11], and SONATA [12]), in order to provide an evolution of the SESAME platform and to "meet" the 5G-PPP Phase-2 requirements, that is to cover the specific network needs of the vertical sectors and their inter-dependencies. 5G ESSENCE enhances the processing capabilities for data that have immediate value beyond locality; it also addresses the processing-intensive small cell management functions, such as Radio Resource Management (RRM)/Self Organising Network (SON) ([13], [14]) and, finally; it culminates with real life demonstrations. For all the above, 5G ESSENCE suggests clear breakthroughs in the research fields of wireless access, network virtualisation, and end-to-end (E2E) service delivery. The existing virtualised resources of small cells [15] will be exploited to their full potential and in a dynamic way, supporting extremely low-latency and the delivery of high-performance services, greater network resiliency, and substantial capacity gains at the access network for the next $5 \mathrm{G}$ stage.

To achieve these important goals, 5G ESSENCE will build upon the SESAME project by developing a distributed edge cloud environment (coined as 'Edge Data Centre" -Edge DC-), based on a two-tier architecture: the first tier (i.e.: Light DC), will remain distributed inside the CESCs for providing latency-sensitive services to users directly from the network's edge. The second tier will be a more centralised, "high-scale" cloud, namely the Main Data Centre (Main DC), which will provide high processing power for computing intensive network applications. It will also have a more centralised view so as to host efficient Quality of Service (QoS) - enabled scheduling algorithms. Both these cloud tiers will form the Edge DC in 5G ESSENCE terminology, which will be viewed as an integrated cloud infrastructure from the upper management and orchestration layers.

On the domain of hardware technologies, the processing power attached to small cells brings new capabilities to the network, as well as new challenges. In addition, the placement of low power/low cost processors to small cells, even with hardware acceleration, will be revised from the perspective of 5G ESSENCE. Although the CESC platform in SESAME is based on non-x86 architectures (ARMv8), the potential use of x86-based, low-cost and low-power processors will also be leveraged due to their efficiency (small form factor, low powered, passively cooled, low price) and their important share in the market.

The research domains mentioned cover only the technical aspects of the proposed 5G ESSENCE activities. However, a significant part of the project is also devoted to the actual demonstration of the outcomes in vertical industries, as they have been identified by 5G-PPP [2]. In order to showcase that $5 \mathrm{G}$ will be able to create a whole new ecosystem for technical and business innovation, 5G ESSENCE unifies computing and storage resources into a programmable and unified small cell infrastructure that can be provided as-a-Service to all related stakeholders. To that end, it provides a clear plan for real life demonstrations in the fields of: (i) multimedia/entertainment; (ii) mission critical communications at emergency events, and; (iii) in-flight connectivity and entertainment. In addition to actual 
demonstrations, 5G ESSENCE is expected to accommodate a much wider range of use cases, especially in terms of ameliorated latency, resilience, coverage and bandwidth. One of its major innovations is that it provides E2E network and cloud infrastructure slices over the same physical infrastructure, in order to fulfil verticalspecific requirements as well as mobile broadband services, in parallel.

\section{The 5G ESSENCE Ecosystem as "Enabler" for Service Deployment}

Network functions are anticipated to take place over a unified operating system in a number of points of presence (PoPs), especially at the edge of the network for fulfilling specific performance targets. As a result, it will heavily rely on emerging technologies such as Software Defined Networking (SDN) [16], Network Functions Virtualization (NFV) [15], Mobile Edge Computing (MEC) [17] and Fog Computing (FC) [18] to achieve the required performance, scalability and agility.

Entering the second phase of 5G-PPP program activities suggests that communication networks become sufficiently flexible to handle a range of applications and services originating from different domains/verticals. At the same time, a transformation towards a significant reduction in cost and the optimal allocation of available resources take the place of initial Key Performance Indicators (KPIs) for driving capacity growth, and coping with the numerous barriers on the infrastructure and management domains. On the users' side, a high-level of personalised services, along with edge mobile capabilities and innovative services are anticipated, since customers require added-value to their choices in order to accommodate specialised requirements with greater quality of both perception and experience.
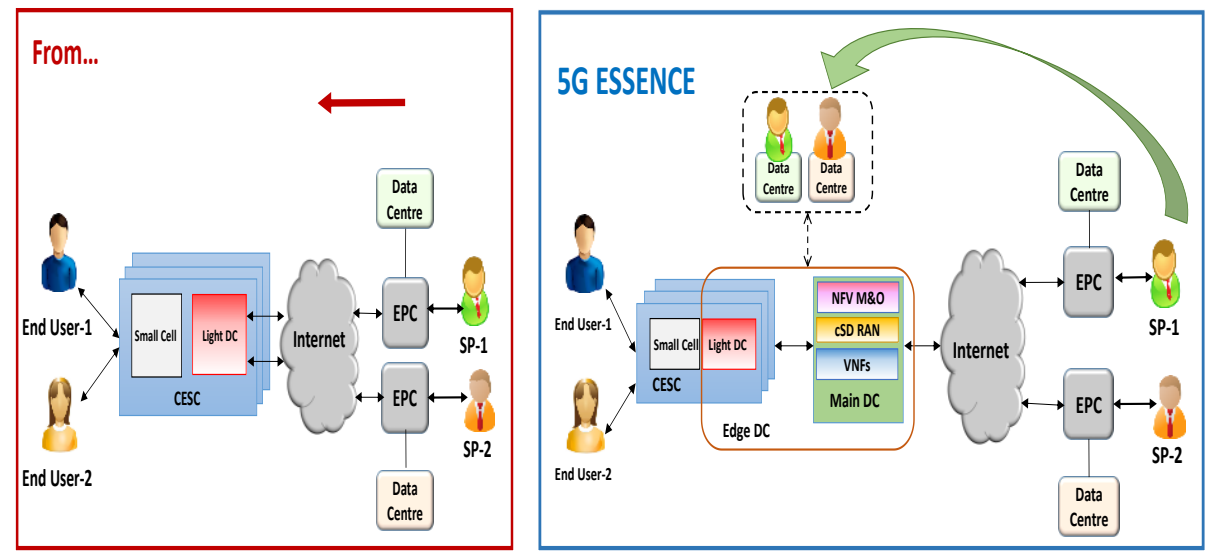

Fig. 1. 5G ESSENCE impact on service deployment.

As the telecom ecosystem moves towards the 5G era, important performance factors such as end-to-end latency critically depend on whether the mobile edge and the target applications reside in an edge cloud located close to the user or not. While 
the air-interface latency can be minimised independently of the service latency, having the application or service functions close to the user is also necessary to reduce the end-to-end round trip time and also the overall service creation time, which again argues for the placement of these functions in a common edge cloud. As shown in Fig.1, the virtualisation of the small cells, as envisaged within the scope of $5 \mathrm{G}$ ESSENCE, supports the inclusion of enhanced mobile-edge computing capabilities that allow acceleration of content, services and applications, increasing responsiveness from the edge of the network. The 5G ESSENCE two-tier cloud resides very close to the users and performs a series of processing-intensive tasks that can neither be achieved with the traditional network infrastructure, nor with the "light" processing power introduced by SESAME which targets to virtualise mainly small cell related software. In this respect, 5G ESSENCE supports an enriched mobile users' experience, minimising service deployment time and, at the same time, it enables network operators and infrastructure owners to open the radio network edge to third-party partners allowing them to rapidly deploy innovative applications and services. The 5G ESSENCE effort "opens the door" to venue owners, e.g., municipalities, stadiums, site owners, and virtually anyone who manages a property and can install and run a local Small Cell network, to deploy a low cost infrastructure and to act as "neutral host network and service provider". Although probably none of such entities would offer static network coverage, many of them could foresee adequate chances for profits generated by exploiting the 5G ESSENCE concepts of multitenant small cells, able to provide wireless network coverage coupled with added-value services in close proximity to customers and visitors that belong to multiple network operators and vertical industries ([20], [21]). In particular, 5G ESSENCE focuses on three real-life use cases associated to vertical industries: (i) 5G edge network acceleration for a stadium, with local video production and distribution; (ii) mission critical applications for public safety (PS) communications providers, and; (iii) next-generation, integrated, in-flight entertainment and connectivity (IFEC) services for passengers.

\section{The Fundamental 5G ESSENCE Architectural Context}

In the 5G ESSENCE approach, the Small Cell concept [22] is evolved as not only to provide multi-operator radio access, but also to achieve an increase in the capacity and the performance of current Radio Access Network (RAN) infrastructures as well as to extend the range of the provided services, while maintaining its agility. To achieve these ambitious goals, the 5G ESSENCE project leverages the paradigms of RAN scheduling and, additionally, it provides an enhanced, edge-based, virtualised execution environment attached to the small cell, taking advantage and reinforcing the concepts of MEC and network slicing [23]. The architecture provided so far by the SESAME project ([7], [8]) acts as a "solid reference point" for 5G ESSENCE. It combines the current $3 \mathrm{GPP}$ framework for network management in RAN sharing scenarios and the ETSI NFV framework for managing virtualised network functions [19]. The CESC offers virtualised computing, storage and radio resources and the CESC cluster is considered as a cloud from the upper layers. This cloud can also be 
"sliced" to enable multi-tenancy. The execution platform is used to support VNFs that implement the different features of the Small Cells as well as to support for the mobile edge applications of the end-users.

Evolving the SESAME-based high-level architecture, the technical approach of 5G ESSENCE is presented in Fig.2, where the working architecture is illustrated with emphasis upon the functional elements and interfaces. As it is depicted, the $5 \mathrm{G}$ ESSENCE architecture allows multiple network operators (tenants) to provide services to their users through a set of CESCs deployed, owned and managed by a third party (i.e., the CESC provider). In this way, operators can extend the capacity of their own 5G RAN in areas where the deployment of their own infrastructure could be expensive and/or inefficient - as it would be the case of, for example, highly dense areas where massive numbers of SCs would be needed to provide the expected services.

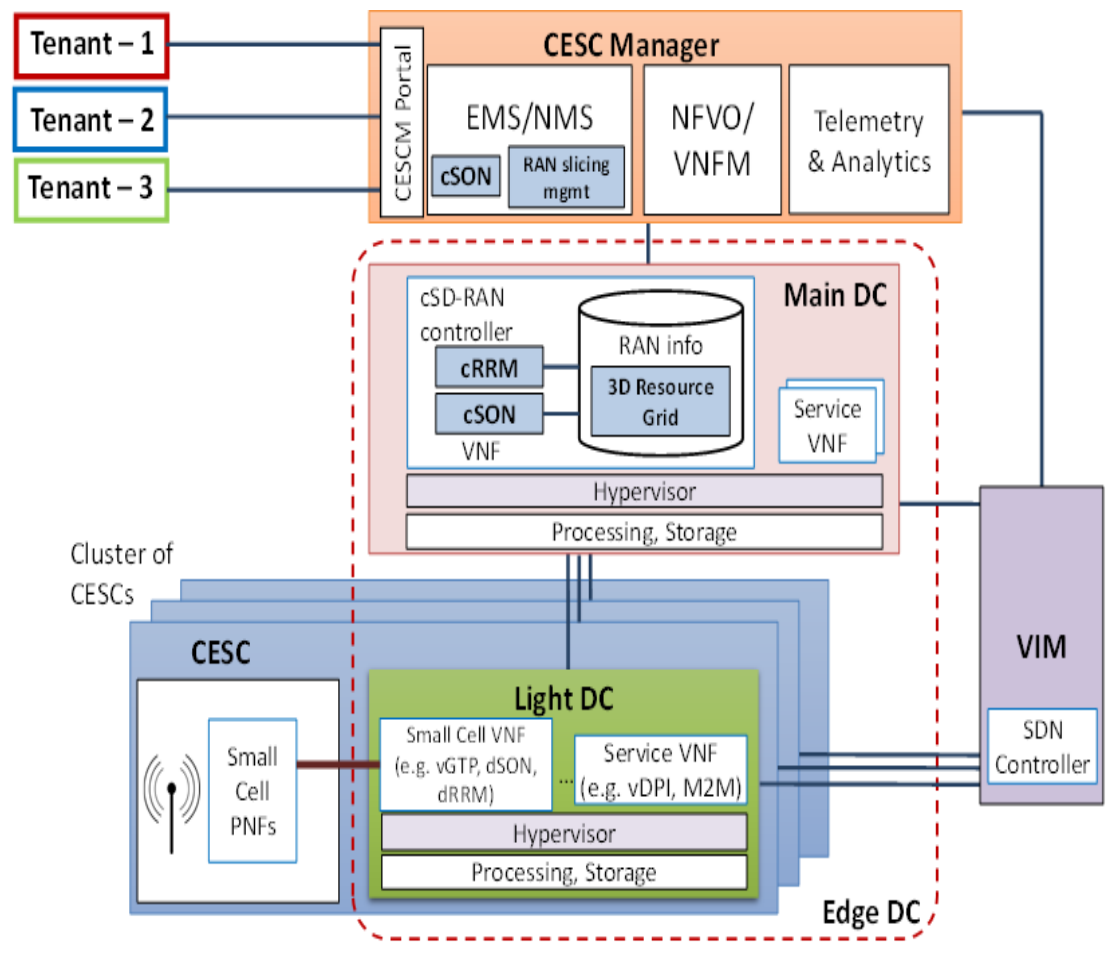

Fig. 2. 5G ESSENCE high-level architecture.

In addition to capacity extension, the 5G ESSENCE platform is equipped with a twotier virtualised execution environment, materialised in the form of the Edge DC, which allows also the provision of MEC capabilities to the mobile operators for enhancing the user experience and the agility in the service delivery. The first tier, i.e., the Light DC hosted inside the CESCs, is used to support the execution of VNFs for carrying out the virtualisation of the Small Cell access. In this regard, network 
functions supporting traffic interception, GTP (GPRS Tunneling Protocol) encapsulation/ decapsulation and some distributed RRM/SON functionalities are expected to be executed therein. VNFs that require low processing power, e.g., a Deep Packet Inspection (DPI), a Machine-to-Machine (M2M) Gateway, and so on, could also be hosted here. The connection between the Small Cell Physical Network Functions (PNFs) and the Small Cell VNFs can be realised through, e.g., the network Functional Application Platform Interface (nFAPI). Finally, backhaul and fronthaul transmission resources will be part of the CESC, allowing for the required connectivity. The second cloud tier, i.e., the Main DC, will be hosting more computation intensive tasks and processes that need to be centralised in order to have a global view of the underlying infrastructure. This encompasses the centralised software-defined RAN (cSD-RAN) controller which will be delivered as a VNF running in the Main DC and makes control plane decisions for all the radio elements in the geographical area of the CESC cluster, including the centralised Radio Resource Management (cRRM) over the entire CESC cluster. Other potential VNFs that could be hosted by the Main DC can also include security applications, traffic engineering, mobility management and, in general, any additional network end-to-end (E2E) services that can be deployed and managed on the 5G ESSENCE virtual networks, effectively and on-demand. The necessary management modules for the operation of the CESC platform and the service provisioning are also depicted in Fig.2, within the CESCM framework. The following subsections provide a more detailed description for each of these architectural components.

The CESC: In our scope, a CESC consists of a Multi-RAT (Radio Access Technology) 5G SC with its standard backhaul interface, standard management connection (TR069 interface for remote management [24]) and with necessary modifications to the data model (TR196 data model [25]) to allow Multi-Operator Core Network (MOCN) radio resource sharing. The CESC will be composed by a physical small cell unit attached to an execution platform based on one of $\mathrm{x} 86$, ARMv8, MIPS64 architectures, to be decided later during the project. Edge cloud computing and networking are realised through the sharing of computation, storage and network resources of those micro-servers present in each CESC and form the Light DC for implementing different features/capabilities of the SC. Therefore, the CESCs becomes a "neutral host" for network operators or virtual network operators that want to share IT and network resources at the edge of the mobile network. The CESC is meant to accommodate multiple operators (tenants) by design, offering Platform-as-a-Service (PaaS), capable of providing the deployed physical infrastructure among multiple network operators. Different VNFs can be hosted in the CESC environment for different tenants. This also provides the support for mobile edge computing applications deployed for each tenant that, operating very near to the end-users, may significantly reduce the service delivery time and deliver composite services in an automated manner [30]. Moreover, the CESC is the termination point of the GTP-User Plane (GTP-U) tunnelling which encapsulates user IP packets from the core network entities (e.g., the Evolved Packet Core (EPC) Serving Gateway (SGW) in LTE) destined to the User Equipment (UE) and vice versa. The CESC exposes different views of the network resources: per-tenant small cell view, and physical small cell substrate, which is managed by the network operator, decoupling the 
management of the virtual small cells from the platform itself. In the CESC, rather than providing multiple S1 (or Iu-h interface) connections from the physical SC to different operators' EPC network elements such as Mobility Management Entity (MME) and SGW, such fan-out is done at the Light DC. The CESC is further the termination of multiple S1 interfaces connecting the CESC to multiple MME/SGW entities as in S1-Flex. The interconnection of many CESCs forms a "cluster" which can facilitate access to a broader geographical area with one or more operators (even virtual ones), extending the range of their provided services, while maintaining the required agility to be able to provide these extensions on demand.

The Edge DC encompassing Main DC and Light DC: $5 \mathrm{G}$ ESSENCE envisages combining the MEC and NFV concepts with SC virtualisation in $5 \mathrm{G}$ networks and enhancing them for supporting multi-tenancy [26]. The purpose of the Edge DC will be to provide Cloud services within the network infrastructure and also to facilitate by promoting and assisting the exploitation of network resource information. To this end, all the normally hardware located modules of the Light DC and the Main DC will be delivered as resources using novel virtualisation techniques. Both networking and computing virtualisation extensions will be developed using open frameworks such as OPNFV. The combination of the proposed Edge DC architecture with the concepts of NFV and SDN will facilitate achieving higher levels of flexibility and scalability. As seen in the detailed architecture in Fig.2, the Main DC will be able to execute different SC and Service VNFs under the control of the CESCM; in particular, the Main DC hosts the cSD-RAN controller which performs cRRM decisions for handling efficiently the heterogeneous access network environment composed of different access technologies (such as 5G RAN, LTE, and Wi-Fi). These radio access networks can be programmable and under the supervision of the centralised controller. The cSD-RAN controller updates and maintains the global network state in the form of a database called as "RAN Information", which includes, among other elements, an abstraction of the available radio resources in the CESC cluster. This abstraction takes the form of a "3D Resource Grid" that characterises the resources in the domains of time/space/frequency. The RAN Information will be used by the cRRM to perform the resource allocation decisions (e.g., scheduling). The cSD-RAN controller can also host centralised SON (cSON) functionalities that need to coordinate multiple small cells, so they are not appropriate for running at the Light DC (for example, this could be the case of InterCell Interference Coordination (ICIC) functions). Other distributed (dSON) functions and/or distributed RRM (dRRM) functions that are of low complexity and that do not involve the coordination of multiple small cells will run at the Light DC. For example, this could be the case of an admission control function that only takes decisions based on the current load existing at a given cell.

The CESCM: Management and orchestration of the proposed uniform virtualised environment, able to support both radio connectivity and edge services, is a challenging task by itself [27]. The management of diverse lightweight virtual resources is of primary importance, enabling a converged cloud-radio environment and efficient placement of services ([28] - [30]). For that purpose, the CESCM shown in Fig. 2 is the central service management and orchestration component in the related 
architecture. Generally speaking, it integrates all the traditional network management elements and the novel recommended functional blocks to realise NFV operations. A single instance of CESCM is able to operate over several CESC clusters at different Points of Presence (PoPs), each constituting an Edge DC through the use of a dedicated VIM (Virtualised Infrastructure Manager) per cluster.

An essential component at the heart of CESCM is the Network Functions Virtualisation Orchestrator (NFVO). It will be in charge of realising network services on the virtualised infrastructure and will include interfaces to interact with the CESC provider for high-level service management (e.g., exchange of network service descriptors and Service level agreements (SLAs) for each tenant). The NFVO composes service chains (constituted by two or more VNF instances located either in one or several CESCs that "jointly" realise a more complex function) and manages the deployment of VNFs over the Edge DC. The NFVO uses the services exposed by the VNF Manager, which will be in charge of the instantiation, update, query, scaling and termination of the VNFs. Moreover, NFVO may include features to enhance the overall system performance, e.g., to improve energy efficiency. The CESCM hosts also the Element Management System (EMS), which provides a package of end-user functions for the management of both the PNFs and VNFs at the CESCs. In particular, the EMS carries out "key" management functionalities such as Fault, Configuration, Accounting, Performance, Security (FCAPS) operations. The EMS will be responsible for partitioning the single whole-cell management view into multiple virtual-cell management views, one per tenant. In this way, a virtualised SC with a set of (limited) management functionalities can be made visible to, e.g., the Network Management System (NMS) of each tenant in order to, for example, collect performance counters, configure neighbour lists for a proper mobility management, etc. It is worth mentioning that, based on the practical lessons learnt from SESAME, all the EMS components of the SESAME architecture (i.e., PNF-EMS, SC-EMS, Service EMS and SLA monitoring of Fig.2), which in practice reveal to be tightly related, will be considered in the 5G ESSENCE architecture under the scope of a single EMS entity. In addition to the NMSs of each tenant, in a general situation, the CESCM can also incorporate a NMS for managing the whole set of CESCs deployed by an operator. This can be appropriate, for example in case that there exist CESCs belonging to different vendors in the same deployment, each one with its own EMS. The EMS/NMS will also host the cSON functionalities (e.g. self-planning, Coverage and Capacity Optimisation (CCO), etc.) and the functionalities for the lifecycle management of RAN slicing (i.e. for the creation, modification or termination of RAN slices). As shown in Fig.2, the CESCM encompasses a telemetry and analytics module that captures and analyses relevant indicators of the network operation. This will provide the CESCM with accurate knowledge models that characterise the behaviour of the network and its users in relation to the utilisation of both cloud and radio resources. This will facilitate the realisation of effective optimisation approaches based on, for example, machine learning (ML) techniques for service placement, which can dynamically adapt to the context of the provided services and their execution environment and to enable automated enforcement of SLAs. Finally, the CESCM also incorporates the CESCM portal. It is a control panel with web Graphical User Interface (GUI) that serves as the "entry point" for the users, both the CESC provider and the tenants, to the CESCM functionalities and constitutes the 
main graphical frontend to access the 5G ESSENCE platform. The CESCM Portal in general provides visual monitoring information of the platform, the agreed SLAs, and the available network services/VNFs, allowing parameters' configuration.

The VIM: The CESCM functions will be built upon the services provided by the VIM for appropriately managing, monitoring and optimising the overall operation of the NFVI (NFV Infrastructure) resources (i.e.: computing, storage and network resources) at the Edge DC. The role of VIM is essential for the deployment of NFV services and to form and provide a layer of NFV resources to be made available to the CESCM functions. The NFV resources will be ultimately offered as a set of application programming interfaces (APIs) that will allow the execution of network services over the decentralised CESCs, located at the edge of the network. As seen in Fig.2, the VIM relies on an SDN controller for interconnecting the VNFs and for offering SFC on the data-plane by establishing the path for the physical connections.

\section{The Fundamental SESAME Architectural Context}

The 5G ESSENCE's goal is the development and demonstration of an innovative architecture, capable of providing Small Cell coverage to multiple operators " $a s$ - $a$ Service", enriched with a two-tier architecture: a first distributed tier for providing low latency services and a second centralised tier for providing high processing power for computing-intensive network applications. To that end, 5G ESSENCE envisages to virtualise and to partition Small Cell capacity while, at the same time, it aims to support enhanced edge cloud services by enriching 5G ESSENCE with an edge cloud. This paper presents a first approach to the high-level overall architecture of the $5 \mathrm{G}$ ESSENCE system. We have initially identified and "positioned" the 5G ESSENCE innovative framework within the modern $5 \mathrm{G}$ era and, furthermore, we have also examined the way how the related ecosystem can enable service deployment, in particular for service vertical-specific needs. However, the core of the present work has been to introduce a proper architectural framework, based on the pillars of network functions virtualisation, mobile-edge computing and cognitive management, to "address" the requirements of a robust and agile network management. In the context of the 5G ESSENCE approach, we have analysed and discussed the main architectural modules as well as their properties, per case, and have identified the framework for further introducing and implementing the system's high-level architecture. The work is based on findings and/or results coming from previous EUfunded research projects (with major contributions coming from the SESAME project) but has been extended and adapted accordingly, to be able to fulfil the requirements coming from the related use cases, associated to vertical industries.

Acknowledgments. This work has been performed in the scope of the 5G ESSENCE European Research Project and has been supported by the Commission of the European Communities (5G-PPP/H2020, Grant Agreement No.761592). 


\section{References}

1. IC Insights, Inc.: IC Market Drivers, A Study of Emerging and Major End-Use Applications Fueling Demand for Integrated Circuits. IC Insights, Inc., Scottsdale, Arizona (2014)

2. European Commission and 5G-PPP: 5G Vision: The 5G-PPP Infrastructure Private Public Partnership: The Next Generation of Communication Network and Services (2015). https://ec.europa.eu/digital-single-market/en/towards-5g

3. European Commission: 5G: Challenges, Research Priorities, and Recommendations - Joint White Paper. European Commission, Strategic Research and Innovation Agenda (2014)

4. Andrews, J.G., Buzzi, S., Choi, W., Hanly, S.V., Lozano, A., Soong, A.C.K., and Zhang, J.C.: What Will 5G Be?, IEEE JSAC, Special issue on 5G Wireless Communications Systems, 32(6), 1065-1082 (2014)

5. Chochliouros, I.P., Sfakianakis, E., Belesioti, M., Spiliopoulou, A.S., and Dardamanis, A.: Challenges for Defining Opportunities for Growth in the 5G Era: The SESAME Conceptual Model. In Proceedings of the EuCNC-2016, pp.1--5 (2016)

6. SESAME ("Small cEllS coordinAtion for Multitenancy and Edge services") 5G-PPP Project, Grant Agreement No.671596. http://www.sesame-h2020-5g-ppp.eu/.

7. Chochliouros, I.P., Giannoulakis I., Kourtis, A., Belesioti, M., Sfakianakis E., Spiliopoulou, A.S., et al:: A Model for an Innovative 5G-oriented Architecture, based on Small Cells Coordination for Multi-Tenancy and Edge Service. In Proceedings of AIAI-2016, IFIP AICT 475, pp.666--675. Springer International Publishing Switzerland (2016)

8. Chochliouros, I.P., Spiliopoulou. A.S., Kostopoulos, A., et al.: Putting Intelligence in the Network Edge through NFV and Cloud computing: The SESAME Approach. In Proceedings of EANN-2017, CCIS 744, pp.1--12. Springer International Publishing (2017)

9. 5G ESSENCE ("Embedded Network Services for 5G Experiences") 5G-PPP Project, Grant Agreement (GA) No.761592. http://www.5g-essence-h2020.eu

10. COHERENT ("Coordinated Control and Spectrum Management for 5G Heterogeneous Radio Access Networks") 5G-PPP Project, GA No.671639. http://www.ict-coherent.eu/.

11. SPEED 5G ("Quality of Service Provision and Capacity Expansion through Extended-DSA for 5G") 5G-PPP Project, Grant Agreement No.671705. https://speed-5g.eu/.

12. SONATA ("Service Programming and Orchestration for Virtualised Software Networks") 5G-PPP Project, Grant Agreement No.671517. http://www.sonata-nfv.eu/.

13. Ramiro, J., and Hamied, K.: Self-Organizing Networks. Self-planning, self-optimization and self-healing for GSM, UMTS and LTE. Wiley, Hoboken (2012)

14. Østerbø, O., and Grøndalen, O.: Benefits of self-organizing networks (SON) for mobile operators. Journal of Computer Networks and Communications 2012, 1-16 (2012)

15. Mosharaf, N.M., Chowdhury, K., and Boutaba, R.: A Survey of Network Virtualization. Computer Networks, 54(5), 862-876, (2010)

16. Haleplidis, E., Salim, J.H., Denazis, S., and Koufopavlou, O.: Towards a Network Abstraction Model for SDN. Journal of Network and Systems Management, 23(2), 309-327 (2015)

17. Fajardo, J.O., Liberal, F., Giannoulakis, I., Kafetzakis, E., Pii, V., Trajkovska, I., Bohnert, T.M., Goratti, L., Riggio, R., et al:: Introducing Mobile Edge Computing Capabilities through Distributed 5G Cloud Enabled Small Cells. Mobile Networks and Applications (MONET), Special Issues on Mobile Networks and Management, 21(4), 564-574 (2016)

18. Vaquero, L.M. and Rodero-Merino L.: Finding your Way in the Fog: Towards a Comprehensive Definition of Fog Computing. ACM SIGCOMM Computer Communication Review, 44(5), 27-32 (2014)

19. European Telecommunications Standards Institute (ETSI): NFV Management and Orchestration - An Overview, GS NFV-MAN 001 v1.1.1. ETSI (2014) 
20. Chochliouros, I.P., Kostopoulos, A., Giannoulakis, I., Spiliopoulou, A.S., Belesioti, M., Sfakianakis, E., Kourtis, A., and Kafetzakis, E.: Using Small Cells from enhancing 5G Networks. In Proceedings of IEEE Conference on Network Function Virtualisation and Software-Defined Networks (NFV-SDN'17), pp.1--6. IEEE (2017)

21. Chochliouros, I.P., Spiliopoulou, A.S., Giannoulakis, I., Spiliopoulou. A.S., et al.: A Novel Architectural Concept for Enhanced 5G Network Facilities. MATEC Web of Conferences 125, 03012 (2017), CSCC-2017, pp.1--7 (2017)

22. Small Cell Forum (SCF): Small Cells and 5G Evolution: A Topic brief. (Document 055.07.01)

http://scf.io/en/documents/055 Small_cells and 5G evolution a topic brief.php

23. Sallent, O., Pérez-Romero, J., Ferrús, R, Augusti, R.: On Radio Access Network Slicing from a Radio Resource Management Perspective. IEEE Wireless Communications Journal 24(5), 166-174 (2017)

24. Broadband Forum (BF): TR-069: CPE WAN Management Protocol (CWMP). BF (2013)

25. Broadband Forum (BF): TR-196v2: Femto Access Point Service Data Model. BF (2017)

26. Giannoulakis, I. Xylouris, G., Kafetzakis, E., Kourtis, A., Fajardo, J.O., Khodashenas, P.S., Albanese, A., Mouratidis, H., and Vassilakis, V.: System architecture and deployment scenarios for SESAME: Small cEllS coordinAtion for Multi-tenancy and Edge services. Proceedings of the IEEE NetSoft 2016 Conference and Workshops, pp.447-452 (2016)

27. Fajardo, J.O., Taboada, Y., and Liberal, F.: Improving Content Delivery Efficiency through Multi-Layer Mobile Edge Adaptation. IEEE Network Management, 29(6) (2015)

28. Blanco, B., Fajardo, J.O., and Liberal, F.: Design of Cognitive Cycles in 5 G Networks. In Proceedings of AIAI-2016, IFIP AICT 475, pp.697--708. Springer International Publishing Switzerland (2016)

29. Kostopoulos, A., Chochliouros, I.P., Kuo, F.-C., Riggio, R., Goratti, L., Nikaein, N., Giannoulakis, I., Perez-Romero, J., Chen, T., Steinert, R., and Panaiotopol, D.: Design Aspects for 5G Architectures. The SESAME and COHERENT approach. In Proceedings of IEEE ICC Workshops 2017, pp.986--992. IEEE (2017)

30. Giannoulakis, I., Kafetzakis, E., Trajkovska, I., Khodashenas, P.S., Chochliouros, I.P., Costa, C., and Bliznakov. P.: The Emergence of Operator-neutral Small Cells as a Strong Case for Cloud-like Computing at the Mobile Edge. Transaction on emerging Telecommunications Technologies, 27(9), 1152-1159 (2016) 